\title{
Simulation and analysis of Hotel optimal guest stream evacuation model in the tourist season Wen Bo
}

(Sichuan Tourism University, Chengdu Sichuan China 610100, )

Keywords: differential evolution; genetic algorithm; optimization model.

\begin{abstract}
During calculation process of hotel guest stream evacuation in the tourist season, the genetic algorithm is used to constantly generate optimal method, based on the theory of "survival of the fittest" to operate unlimited reproduction for the better method, which makes the algorithm premature convergence, evacuation method trapped in a local optimum, cannot achieve the purpose of evacuating the overall congested traffic area. Therefore, optimal hotel guest flow evacuation method in the tourist season is proposed based on differential evolution algorithm. The guest in congestion Hotel guest flow region is regarded as separate particles, initialization is processed for the initial velocity and initial position of particle, so as to obtain change range of particle position and velocity. Then the differential evolution algorithm is adopted for precocious particle, obtain the diversity of particles, and avoid premature convergence of the population. Finally, the differential evolution algorithm is utilized for optimizing continuous variable function of guests in hotel guest flow concentrated region, so as to realize the evacuation of Hotel guest flow concentrated region. Simulation results show that, the proposed differential evolution genetic algorithm can not only evacuate guest concentrated area in the tourist season, meet all the requirements, but also in modified process of evacuation algorithm, can increase the diversity of population, and get better evacuation plans.
\end{abstract}

\section{Introduction}

With the rapid development of tourism, tourist safety has also attracted more and more attention [1]. In the course of travelling, how to ensure the timely evacuation of the guest flow in the hotel when emergency appears, in the tourist season, which is the problem must be solved in tourist industry [2-4]. To this end, simulation is necessary for modelling optimal hotel guest flow evacuation in the tourist season. Only the safety of tourists in the hotel is guaranteed, the tourists travel safety can be guaranteed, thus conducive to the sustainable development of tourism industry [5-7]. Therefore, optimal hotel guest flow evacuation simulation in the tourist season, has become the focus of the tourism industry, also has been widespread concerned, have good development space and the huge development potential of [8-10].

\section{2 the principle of hotel guest flow evacuation method based on differential evolution algorithm in the tourist season}

In the modeling process of optimal hotel guest flow evacuation model in tourist season with differential evolution algorithm, the guest is regarded as particles, initialization is processed for the initial velocity and initial position of particle firstly. Then the differential evolution algorithm is adopted for precocious particle, obtain the diversity of particles, and avoid premature convergence of the population. Finally, the differential evolution algorithm is utilized for optimizing continuous variable function of guests in hotel guest flow concentrated region, so as to realize the evacuation of hotel guest flow. The detailed principle as follows:

2.1 initialization operation

In the process of utilizing differential algorithm for hotel guest flow evacuation, guests in the hotel will be regarded as a separate particle, first of all, it need to be initialized. Specific methods are as follows:

Assuming the space dimension of evacuation problem can be described by $D$, the population 
size of particle swarm can be described by $N$, the change range of hotel guests in the $j$ dimension is $\left[a_{j}, b_{j}\right]$. In space dimension $D$, a random initialization vector $y_{0}=\left[y_{0,1}, \cdots, y_{0, j}, \cdots, y_{0, D}\right]$ is generated $y_{0, j} \neq 0.25,0.5,0.75$, between each dimension of $y_{0}$ have differences. The vector $y_{0}$ as the initial value of iterative processing, the following formula is utilized to describe particle vector sequence ${ }^{y_{n+1, j}}$ :

$$
\begin{gathered}
y_{n+1, j}=f\left(\mu, y_{n, j}\right)=\mu y_{n, j}\left(1-y_{n, j}\right) \\
(n=0,1,2, \cdots ; j=1,2, \cdots D)
\end{gathered}
$$

Among them, the range of particle vector sequence is $\left[a_{j}, b_{j}\right]$, the following formula is utilized to describe the mapping value of feasible solution ${ }^{x_{n+1, j}}$ :

$$
x_{n+1, j}=a_{j}+\left(b_{j}-a_{j}\right) \times y_{n+1, j}
$$

If $j=1,2, \cdots D, D$ mapping values can be obtained, the vector $x_{n+1}=\left(x_{n+1,1}, x_{n+1,2}, \cdots, x_{n+1, D}\right)$ constituted of the mapping vector is the feasible solution in optimal hotel guest flow evacuation problem, which is used to describe the location of the hotel guests. Every guest in hotel have a position, then the position of $N$ hotel guests is obtained, namely to the position of $N$ particles, the initial position of these particles is the initial population of particle algorithm. By using the same method, the speed of the particle is initialized, according to the initialization results, speed change range of particles is $\left[V_{\min }, V_{\max }\right]$.

\section{2 judgment method of population premature}

Assuming number of particles in particle swarm can be described by $N, f_{i}$ is used to describe the fitness of $i$ particle. $f_{\text {avg }}$ used to describe average fitness value of all the particles, the following formula is utilized to describe particle swarm fitness variance:

$$
\sigma^{2}=\frac{1}{N} \sum_{i=1}^{N}\left(\frac{f_{i}-f_{\text {avg }}}{f}\right)^{2}
$$

In the formula, $f$ is used to describe the normalization coefficient, the magnitude of $\sigma^{2}$ can be restricted. The value range of $f$ is not restricted, but need to meet the following conditions:

The particle swarm is normalized, the maximum value of $\frac{\left|f_{i}-f_{\text {avg }}\right|}{f}$ should be less than 1; $f$ changes with the times of iterative evolution,

$$
f_{\text {avg }}=\frac{1}{N} \sum_{i=1}^{N} f_{i}
$$

. The following formula is used to calculate the value of $f$ :

$$
f=\left\{\begin{array}{cl}
\max _{1 \leq i \leq N}\left|f_{i}-f_{\text {avg }}\right| & \text { if } \max _{1 \leq i \leq N}\left|f_{i}-f_{\text {avg }}\right|>1 \\
1 & \text { otherwise }
\end{array}\right.
$$

Fitness variance $\sigma^{2}$ can reflect the aggregation degree of hotel guest flow in the tourist season, namely diversity of particle population. The bigger the $\sigma^{2}$ is, the aggregation degree is lower, the diversity of the population is better. The smaller the $\sigma^{2}$ is, the aggregation degree is higher, the diversity of the population becomes worse, it is easy to fall into local optimum. Therefore, the following method is adopted to determine particle prematurity: assuming that the particle population fitness variance $\sigma^{2}<\sigma_{T}^{2}$, indicates that the population is precocious. Among them, $\sigma_{T}^{2}$ is used to describe the predetermined threshold of adaptation variance. On the other hand, assuming that adaptation variance of the particle population is $\sigma^{2}>\sigma_{T}^{2}$, then the population isn't precocious.

2.3 The process of differential evolution operation

The detailed steps of differential evolution are as follows: 
(1) initialize operation for the related parameters of particles in population, like velocity and position. According to the hotel guest evacuation problem in tourist season, the dimension of guest flow is $D$, the number of guests in the hotel is $N$, the maximum moving speed during evacuation process of the guests is $V_{\max }$, learning factor $c_{1}$ and $c_{2}$, the default number of iterative processing $T$; the initial position $x=\left(x_{1}, x_{2}, \cdots, x_{D}\right)$ and velocity $v=\left(v_{1}, v_{2}, \cdots, v_{D}\right)$ of particles are calculated; and setting $t=1$;

(2) for fitness of all particles in the population is calculated. The extremum pbest of individual particle and the global extremum ${ }^{\text {gbest }}$ of population are calculated;

(3) the speed and position of particles in the population are updated. Assuming speed and position of particles have reached the set range, then the maximum value is obtained.

(4) the fitness variance $\sigma^{2}$ of the population is calculated, so as to realize the particle precocity judgment, assuming caught early, go to step (5), otherwise go to step (6).

(5) the differential evolution is conducted to particle.

(6) iterative algorithm terminates condition judgment. If $t>T$, then stop the operation, the optimal solution is obtained; otherwise go to step 2, and set $t=t+1$.

2.4 The implementation of optimal hotel guest flow evacuation in tourist season

Each guest in optimal hotel guest flow evacuation process during tourist season can be viewed as a variable, therefore, with the differential evolution algorithm can optimize continuous variable function of hotel guest flow. The initial population of hotel guest flow in tourist season is $X^{0}=\left\{X_{1}^{0}, X_{2}^{0}, \ldots, X_{D}^{0}\right\}$, the $G$ generation population can be described as $X^{G}=\left\{X_{1}^{G}, X_{2}^{G}, \ldots, X_{D}^{G}\right\}$, the number of guests in population is $N_{N P}$, the coefficient of individual variation is $F$, cross probability between the individual is $P_{C R}$. Then the differential evolution algorithm can be divided into the following 4 steps:

(1) the population initialization processing. The initial population of hotel guest flow is $X^{0}$, the population size is $N_{N P}$, the scaling factor is $F$, the crossover probability is $P_{C R}$.

(2) individual mutation process. $r_{1}, r_{2}, r_{3} \in \operatorname{rand}(1 . n)$ is randomly selected in a population, and $r_{1} \neq r_{2} \neq r_{3} \neq i$, then:

$\mathrm{u}_{i}^{G}=x_{r 1}^{G}+F \chi\left(x_{r 2}^{G}-x_{r 3}^{G}\right)$

Among them, $G$ is used to describe the evolution times, $x_{r 1}^{G}$ is used to describe the parent vector; $\left(x_{r 2}^{G}-x_{r 3}^{G}\right)$ is used to describe the father generation difference vector.

(3) individual crossover operation treatment. Two individuals are selected arbitrarily in the population, cross operation is applied, its formula is as following:

$\mathrm{W}_{i}^{G}=\left\{\begin{array}{c}u_{i j}^{G}, \operatorname{rand}[0,1]<P_{C R}, \text { or, } \mathrm{j}=\mathrm{j}_{\text {rand }} \\ x_{i P}^{G}, \operatorname{rand}[0.1] \geq P_{C R} \text { or } \mathrm{j} \neq \mathrm{j}_{\text {rand }}\end{array}\right.$

(4) selection of operation process. After cross operation is applied for individuals in a population, evacuation route is selected using the following formula:

$X_{i}^{G+1}=\left\{\begin{array}{l}W_{i}^{G}, f\left(W_{i}^{G}\right) \leq f\left(X_{i}^{G}\right) \\ X_{i}^{G}, f\left(W_{i}^{G}\right)>f\left(X_{i}^{G}\right)\end{array}\right.$

Continue to repeat the above steps, until obtains the optimal solution, or reaches a preset number of iterative processing.

\section{3 simulation experiments}

The experiment tested in the optimization calculation from several aspects, like convergence 
speed, convergence rate, average value and stability of algorithm.

The experimental environment and the parameter setting. The hardware environment is the Intel Core Duo 2.20GHz CPU, 2GB memory RAM, software environment is WIN XP system. The programming language is MATLAB.

Table 1 Comparison of the performance of various optimization algorithm

\begin{tabular}{|c|c|c|c|c|}
\hline Algorithm & $\begin{array}{c}\text { Speed of } \\
\text { convergence }\end{array}$ & $\begin{array}{c}\text { rate of } \\
\text { convergence }(\%)\end{array}$ & AVG & Stability \\
\hline DE & 32121 & 92 & 6.88 & $6.1 \mathrm{e}$ \\
\hline ISDE & 26412 & 100 & 5.23 & $5.2 \mathrm{e}$ \\
\hline MEDE & 31864 & 100 & 4.19 & 4.1e \\
\hline QAISDE & 11264 & 100 & 4.25 & $2.8 \mathrm{e}$ \\
\hline $\begin{array}{l}\text { Improved } \\
\text { algorithm }\end{array}$ & 3392 & 100 & 3.26 & 1.6e \\
\hline $\begin{array}{l}\text { In Table } \\
\text { independent } \\
\text { error requir } \\
\text { around thre } \\
\text { optimizatior } \\
\text { actual needs }\end{array}$ & $\begin{array}{l}\text { e convergenc } \\
\text { ation } 30 \text { time } \\
\text { s. The impro } \\
\text { isand times, } \\
\text { rithm need to } \\
\text { affic congesti }\end{array}$ & $\begin{array}{l}\text { eed represents the } \\
\text { optimization algori } \\
\text { algorithm can obtair } \\
\text { h can satisfy the act } \\
\text { ulate for tens of tho } \\
\text { vacuation. }\end{array}$ & $\begin{array}{l}\text { value } \\
\text { search } \\
\text { ffic co } \\
\text { cuatior } \\
\text { times, }\end{array}$ & $\begin{array}{l}\text { algorithm when } \\
\text { l strategy meets } \\
\text { ation strategy at } \\
\text { while the other } \\
\text { cannot meet the }\end{array}$ \\
\hline
\end{tabular}

\section{Conclusion}

During calculation process of hotel guest stream evacuation in the tourist season, the genetic algorithm is used to constantly generate optimal method, based on the theory of "survival of the fittest" to operate unlimited reproduction for the better method, which makes the algorithm premature convergence, evacuation method trapped in a local optimum, cannot achieve the purpose of evacuating the overall congested traffic area. Therefore, optimal hotel guest flow evacuation method in the tourist season is proposed based on differential evolution algorithm. The guest in congestion Hotel guest flow region is regarded as separate particles, initialization is processed for the initial velocity and initial position of particle, so as to obtain change range of particle position and velocity. Then the differential evolution algorithm is adopted for precocious particle, obtain the diversity of particles, and avoid premature convergence of the population. Finally, the differential evolution algorithm is utilized for optimizing continuous variable function of guests in hotel guest flow concentrated region, so as to realize the evacuation of Hotel guest flow concentrated region. Simulation results show that, the proposed differential evolution genetic algorithm can not only evacuate guest concentrated area in the tourist season, meet all the requirements, but also in modified process of evacuation algorithm, can increase the diversity of population, and get better evacuation plans.

\section{References}

[1] Jiang Hui, Sun Qingfeng. Overview of the methods of emergency evacuation [J], science and technology information, 2013.2:215.

[2] Ma Yuhe, Sun Jinxiang. On evacuation induction [J]. China public safety, 2004.1:167.

[3] Wang Lei, Wang Fang. Discussion on the safety evacuation problems of construction engineering $[\mathrm{J}]$. Small and medium-sized enterprises management and technology, 2009.3:251-252.

[4] Ying Bingxiang, Hu Dianlin. Discussion on the base construction of the population evacuation [J]. Anshan social science, 2010.1:28-29.

[5] Fang Zheng, Lu Zhaoming. Grid model of building refuge and evacuation [J]. Chinese Safety Science Journal, 2001.4:10-13.

[6] Hou Yucheng, Liu Yubao, Mei Zhibin, Wu Xiaochuan. The evacuation route generation technology of intelligent evacuation indicating system [J]. Fire science and technology, 
2014.1:89-91.

[7] Li Huaizhong, Guo Qunying, Chen Jun, Liu Junfeng. Emergency evacuation and emergency escape [J]. Technology supervision in petroleum industry, 2010.12: 22-24.

[8] Yang Qingxi. Building safety evacuation system design [J]. Journal of Zaozhuang University, 2014.5:140-142.

[9] Wang Jun. Safety evacuation of public building construction [J]. Journal of Wuhu Institute of Technology, 2007.3:108-109.

[10] Zhang Dongping. Discussion on safety evacuation of high-rise building [J]. Government legality, 2007.05S:28. 